\title{
Polymerized hybrid perovskites with enhanced stability, flexibility and lattice rigidity
}

\section{Wenjing Chen}

University of Science and Technology of China

\section{Yongliang Shi}

University of Science and Technology of China

Jia Chen

University of Science and Technology of China

\section{Pingchuan $\mathrm{Ma}$}

University of Science and Technology of China

\section{Zhibin Fang}

University of Science and Technology of China

\section{Dan Ye}

University of Science and Technology of China

\section{Yiyang Lu}

University of Science and Technology of China

\section{Yongbo Yuan}

Central South University https://orcid.org/0000-0002-4606-4611

Jin Zhao

University of Science and Technology of China https://orcid.org/0000-0003-1346-5280

Zhengguo Xiao ( $\nabla$ zhengguo@ustc.edu.cn )

University of Science and Technology of China https://orcid.org/0000-0001-6646-1166

\section{Article}

Keywords: organometal halide perovskites, soft lattice, lattice rigidity

Posted Date: January 19th, 2021

DOI: https://doi.org/10.21203/rs.3.rs-129636/v1

License: (9) (1) This work is licensed under a Creative Commons Attribution 4.0 International License. Read Full License 
Version of Record: A version of this preprint was published at Advanced Materials on September 30th, 2021. See the published version at https://doi.org/10.1002/adma.202104842. 
Polymerized hybrid perovskites with enhanced stability, flexibility and lattice rigidity Wenjing Chen ${ }^{1 \dagger}$, Yongliang Shi ${ }^{1 \dagger}$, Jia Chen ${ }^{1}$, Pingchuan Ma ${ }^{1}$, Zhibin Fang ${ }^{1}$, Dan Ye ${ }^{1}$, Yiyang $\mathrm{Lu}^{1}$, Yongbo Yuan ${ }^{2}$, Jin Zhao ${ }^{1}$, Zhengguo Xiao ${ }^{1 *}$

${ }^{1}$ Hefei National Laboratory for Physical Sciences at the Microscale, CAS Key Laboratory of Strongly-coupled Quantum Matter Physics, Department of Physics, University of Science and Technology of China, Hefei, Anhui 230026, China

${ }^{2}$ Hunan Key Laboratory of Supermicrostructure and Ultrafast Process, School of Physics and Electronics, Central South University, Changsha, Hunan 410083, China

†These authors contribute equally to this work *Correspond to: zhengguo@ustc.edu.cn

Abstract: The intrinsic soft lattice nature of organometal halide perovskites (OHPs) makes them very tolerant to defects and ideal candidates for solution-processed optoelectronic devices. However, the soft lattice results in low stability towards external stresses such as heating and humidity, and induces high density of phonons, causing strong electron-phonon coupling. Here, we report solid-state polymerization of OHPs using unsaturated 4-vinylbenzylammonium as organoammonium cations without damaging perovskite structure and its tolerance to defects. The polymerized perovskites show enhanced stability and flexibility compared to regular three-dimensional and twodimensional perovskites. Furthermore, the polymerized 4-vinylbenzylammonium group improves perovskite lattice rigidity substantially, resulting in reduced electron-phonon coupling and non-radiative recombination rate, and enhanced carrier mobility because of suppressed phonon scattering. We demonstrate efficient polymerized perovskite based light-emitting diodes with an external quantum efficiency of $23.2 \%$ and enhanced operation stability. 


\section{Introduction}

27 Organometal halide perovskites (OHPs), including three-dimensional (3D) and two28 dimensional (2D) perovskite (Ruddlesden-Popper (RP) and Dion-Jacobson (DJ) phases), have become as a new generation of semiconducting materials for electronic and optoelectronic devices $^{1-4}$. An intrinsic nature of OHPs is its soft lattice due to weak ionic bonding between metal and halide, and non-covalent bonding between organic cations and the inorganic framework $^{5}$. The soft lattice nature makes OHPs very tolerant to defects compared to conventional inorganic semiconductors such as silicon and III-V compounds ${ }^{6-8}$, making OHPs ideal candidates for solution-processed optoelectronic devices. However, the soft lattice results in low stability towards external stresses such as heating and humidity ${ }^{7,9}$. In addition, it induces high density of phonons, causing strong electron-phonon coupling (EPC) in $\mathrm{OHPs}^{10,11}$, particularly in 2D RP phase with weak Van der Waals force between adjacent organic layers ${ }^{12-}$ 14. The strong EPC results in rapid non-radiative exciton quenching, and low photoluminescence quantum yield (PLQY) ${ }^{14-16}$, which is detrimental to devices working at high temperature or under high current density. For example, perovskite light-emitting diodes (PeLEDs) based on 2D OHPs, such as phenethylammonium lead iodide, has electroluminescence only at liquid nitrogen temperature ${ }^{17}$.

There are many small ligands and polymer ligands explored to improve the stability and/or optoelectronic properties of OHPs, such as those containing $\mathrm{C}=\mathrm{O}$ group ${ }^{18-21}$ and hydrophobic group $^{22}$. Their functions include trap passivation ${ }^{19,20}$, making the film more hydrophobic ${ }^{21,22}$, improving the film quality ${ }^{21,23}$, and/or suppression of ion migration ${ }^{18}$, etc. (Supplementary 
(Supplementary Fig. 1), and form polymer-perovskite mixture, rather than in the perovskite lattice ${ }^{18-22}$. A limitation of those approaches is that the polymer additives cannot change the intrinsic properties of OHPs such as their strong EPC, although they can form coordinate bonding with the metallic lead or dangling bonds of halides at the crystal surface.

In this work, we report solid-state polymerization (SSP) of perovskite lattice using unsaturated organoammonium cations 4-vinylbenzylammonium (VBA) without damaging the perovskite structure. The polymerized perovskite, a new phase of OHPs which is different from RP or DJ phase, shows polymer-like behavior such as enhanced water/thermal stability and flexibility, resulted from the high molecular weight of the polymerized-VBA (PVBA) ligands and strong covalent $\mathrm{C}-\mathrm{C}$ bonding between adjacent organoammonium groups. More excitingly, as organic component of the perovskite structure which is superior to regular polymer additives, the PVBA ligands increase rigidity of perovskite lattice without scarifying its tolerance to defects, resulting in less activated optical phonon modes and weakened EPC, suppressed nonradiative exciton recombination rate, as well as increased electron/hole mobility due to less phonon scattering. Benefitted from the above advantages of polymerized perovskite, highperformance PeLEDs with a record external quantum efficiency (EQE) of $23.2 \%$ and improved operation stability was achieved.

\section{Theoretical prediction of the formation of polymerized perovskite.}

SSP within the organic component of the perovskite structure depends highly on the molecular structure of organoammonium ligands ${ }^{24,25}$. Firstly, the distance between two adjacent $\mathrm{C}=\mathrm{C}$ functional groups in the perovskite lattice before polymerization should be less than $5 \AA$ to activate $\mathrm{SSP}^{25}$. Secondly, the distance between two adjacent ammonium ligands $\left(\mathrm{d}_{\mathrm{N}-\mathrm{N}}\right)$ after 
polymerization should match the distance between two adjacent metal-halide octahedral $\left(\mathrm{d}_{\mathrm{M}}\right.$ м). Lastly, ammonium ligands should be susceptible to polymerization without additional initiators. VBA has a similar structure with phenymethylammonium (PMA), a very popular ligand in 2D perovskites, but has an unsaturated vinyl group in para position of the aromatic phenyl ring (Fig. 1a). The styrene group in VBA monomer enables easy polymerization under

UV irradiation or low-temperature heating ${ }^{26,27}$. Therefore, we select VBA monomer as the organoammoniums for SSP. As shown in Fig. 1a, the styrene groups are first excited to a singlet state by UV irradiation or heating ${ }^{27}$, followed by intersystem crossing to a triplet state and formation of radicals on the vinyl carbons. The radicals then form new covalent bonds with other nearby vinyl groups ${ }^{26}$, forming PVBA ligands.

We first estimated the feasibility of forming polymerized perovskite phase with VBA monomer and their molecular configuration before and after SSP using density functional theory (DFT) (see Methods). Fig. 1b and Supplementary Fig. 2a show the calculated molecule structure of $(\mathrm{VBA})_{2} \mathrm{PbI}_{4}$ perovskite with VBA before polymerization, which is similar with that of $(\mathrm{PMA})_{2} \mathrm{PbI}_{4}{ }^{28}$. The distances between adjacent unsaturated $\mathrm{C}=\mathrm{C}$ bonds are 3.8 and $4.1 \AA$ (Fig. 1c), satisfying the criteria for the SSP. Our calculation shows that the Gibbs free energy of $(\mathrm{VBA})_{2} \mathrm{PbI}_{4}$ decreases by $3.6 \mathrm{eV}$ per unit after SSP, indicating easy formation and stabilization of polymerized-(VBA) ${ }_{2} \mathrm{PbI}_{4}$ (noted as $\left.(\mathrm{PVBA})_{2} \mathrm{PbI}_{4}\right)$. Fig. 1d and Supplementary Fig. 2b show a molecular structure of (PVBA) $)_{2} \mathrm{PbI}_{4}$, which has the lowest Gibbs free energy. The $\mathrm{d}_{\mathrm{N}-\mathrm{N}}$ in the (PVBA) ${ }_{2} \mathrm{PbI}_{4}$ structure varies from 5.3 to $7.7 \AA$ depending on tilting angle of the aromatic phenyl ring (Fig. 1e), matching $\mathrm{d}_{\mathrm{M}-\mathrm{M}}$ of methylammonium lead iodide/bromide/chloride (6.3 $\AA, 5.9 \AA, 5.7 \AA$, respectively $)^{29}$. 

lattice, as predicted by DFT calculation. For example, the distance between two inorganic layers reduces from $18.3 \AA$ to $17.2 \AA$ due to formation of strong covalent bonding between two adjacent VBA layers (Fig. 1b,d). The orientations of phenyl rings in adjacent VBA layers change from vertical to parallel after SSP, in order to minimize their repulsion interaction. The average $\mathrm{Pb}-\mathrm{I}-\mathrm{Pb}$ angles also slightly increase from $148.1^{\circ}$ to $152.7^{\circ}$ to adapt to the configuration of PVBA (Fig. 1f). As a result of the greater $\mathrm{Pb} s$ and I $\mathrm{p}$ orbital overlap, the bandgap of (VBA) ${ }_{2} \mathrm{PbI}_{4}$ is reduced by $0.06 \mathrm{eV}$ after SSP (Supplementary Fig. 2). Nevertheless, regardless of those above minor configuration changes, the $(\mathrm{VBA})_{2} \mathrm{PbI}_{4}$ retain its perovskite structure after SSP. dimethylformamide (DMF) to prepare perovskite films. Interestingly, the mixed $\mathrm{VBAI}-\mathrm{PbI}_{2}$ minor structural and bandgap changes after SSP, as predicted by DFT calculation, were also experimentally observed. As shown in Fig. 2a,b, both absorption edge and PL peak slightly 
redshifts, and d-spacing of $(002)$ plane $\left(\mathrm{d}_{(002)}\right)$ gradually decreases from $18.1 \AA$ to $17.2 \AA$.

Fourier transform infrared spectroscopy (FTIR), proton nuclear magnetic resonance $\left({ }^{1} \mathrm{H}\right.$ NMR) were used to track the polymerization process of VBA monomers in the $2 \mathrm{D}(\mathrm{VBA})_{2} \mathrm{PbI}_{4}$ perovskite structure. As shown in Fig. $2 \mathrm{c}$, the $\mathrm{C}=\mathrm{C}$ stretching, and $=\mathrm{CH}_{2}$ and $=\mathrm{CH}$ wagging vibration peaks from vinyl group gradually disappear with UV exposure time increasing from 10 to 60 min, indicating the polymerization of VBA monomers in the $(\mathrm{VBA})_{2} \mathrm{PbI}_{4}$ films. The ${ }^{1} \mathrm{H}$ NMR peaks assigned to the vinyl group decrease obviously with the appearance of new peaks in the $\mathrm{sp}^{3}$ carbon region at $2.5 \mathrm{ppm}$, resulted from polymerization of vinyl group (Fig. 2d). In addition, the peaks come from aromatic proton are partially shifted to around $7.0 \mathrm{ppm}$ due to the shielding effect caused by polymerization. By normalizing the known quantity of tetramethylsilane (TMS, as the internal standard material), the percentage of polymerized VBA monomers can be calculated by comparing the integral area of $\mathrm{a}, \mathrm{b}$, and $\mathrm{c}$ peaks before and after polymerization. It is shown that around $65 \%$ of VBA monomers are polymerized after UV for $60 \mathrm{~min}$. Gel permeation chromatography (GPC) was conducted to investigate the degree of polymerization (DP) of VBA monomers in (PVBA) $2 \mathrm{PbI}_{4}$ films. As shown in Fig. 2e, the average molecular weight $\left(\mathrm{M}_{\mathrm{n}}\right)$ of PVBA is positively correlated with the UV exposure time with DP ranging from 4 to 75.

In addition to ( $\mathrm{PVBA})_{2} \mathrm{PbI}_{4}$ perovskite, a series of quasi-2D perovskite films with different dimensions (PVBA) ${ }_{2} \mathrm{MA}_{\mathrm{n}-1} \mathrm{~Pb}_{\mathrm{n}} \mathrm{I}_{3 \mathrm{n}+1}$ were also successfully prepared. They all show characteristics of quasi-2D perovskites in absorption, PL, as well as XRD (Supplementary Fig. 5). The d-spacing of (PVBA) $)_{2} \mathrm{MA}_{n-1} \mathrm{~Pb}_{n} \mathrm{I}_{3 \mathrm{n}+1}$ films calculated from (001) peak of XRD match well with the high-resolution transmission electron microscopy (HRTEM) images and DFT 
calculations (Supplementary Fig. 6), demonstrating the formation of (PVBA) ${ }_{2} \mathrm{MA}_{\mathrm{n}-1} \mathrm{~Pb}_{\mathrm{n}} \mathrm{I}_{3 \mathrm{n}+1}$

137 perovskite structure.

138 The polymerized perovskite with enhanced stability.

139 A very attractive property of polymerized perovskite is its enhanced intrinsic stability.

140 Thermogravimetric analysis (TGA) was used to investigate the thermal stability of the

141 (PVBA) $)_{2} \mathrm{PbI}_{4}$ film. As shown in Fig. 2f, three stages of TGA curves are observed, which can

142 be attributed to loss of VBA capping layer at the surface, organic amine salt in the perovskite

143 framework, and $\mathrm{PbI}_{2}$, respectively ${ }^{30}$. An obvious delay is observed in the organic species of

$144(\mathrm{VBA})_{2} \mathrm{PbI}_{4}$ after UV treatment, suggesting better thermal stability after polymerization. The

145 improved thermal stability can be attributed to the strong covalent bond between

146 organoammonium ligands between adjacent layers, and higher molecular weight of PVBA than

147 VBA monomer.

148 The polymerized perovskite $(\mathrm{PVBA})_{2} \mathrm{PbI}_{4}$ also has much improved humid stability. We 149 directly soaked both (VBA) ${ }_{2} \mathrm{PbI}_{4}$ and (PVBA) ${ }_{2} \mathrm{PbI}_{4}$ films in water without any encapsulation

150 (Supplementary Video 1). Fig. 2g shows the photographs of the films immersed in water for

151 different time. The control film degrades rapidly in seconds, while the polymerized perovskite

152 (PVBA $)_{2} \mathrm{PbI}_{4}$ film remain unchanged for $30 \mathrm{~s}$. In addition to the above reasons for enhanced

153 thermal stability, the improved humid stability may also benefit from the increased 154 hydrophobicity of PVBA.

155 Benefited from its polymer-like behavior, the polymerized perovskite shows greatly 156 enhanced flexibility. Fig. 2h shows scanning electron microscopy (SEM) images of the 157 (VBA) $)_{2} \mathrm{PbI}_{4}$ and $(\mathrm{PVBA})_{2} \mathrm{PbI}_{4}$ films (thickness $500 \mathrm{~nm}$ ) upon 500 bending cycles with a 
bending radius of $3 \mathrm{~mm}$. The $(\mathrm{VBA})_{2} \mathrm{PbI}_{4}$ film shows many cracks while the polymerized

159

160

161

162

163

164

165

166

167

168

169

170

171

172

173

174

175

176

177

178

179 perovskite (PVBA) $)_{2} \mathrm{PbI}_{4}$ remains very compact, demonstrating its potential in flexible devices.

\section{The polymerized perovskite with enhanced lattice rigidity.}

Another exciting property of polymerized perovskite is its enhanced lattice rigidity because of the polymerized carbon chains in the lattice. This is in direct contrast with the polymerperovskite mixture film with polymers at the gain boundary ${ }^{18,21}$. We first calculated average atomic displacement of ( $\mathrm{PVBA})_{2} \mathrm{PbI}_{4}$, and $(\mathrm{PMA})_{2} \mathrm{PbI}_{4}$ as control, at different temperatures using DFT (see Methods). As shown in Fig. 3a, the root mean squared displacement (RMSD) of carbon atoms in (PVBA) $)_{2} \mathrm{PbI}_{4}$ is a half of that of control (PMA) $)_{2} \mathrm{PbI}_{4}$ structure due to the restricted motions of carbon atoms after polymerization. Notably, resulted from the interaction between organic and inorganic components in the perovskite structure, the RMSD of both $\mathrm{Pb}$ and $\mathrm{I}$ atoms are also smaller in (PVBA) $)_{2} \mathrm{PbI}_{4}$ as temperature increasing from $80 \mathrm{~K}$ to $380 \mathrm{~K}$. Fig. $3 \mathrm{~b}$ shows the DFT calculated real-space displacement of both $\left(\mathrm{PVBA}_{2} \mathrm{PbI}_{4}\right.$ and $(\mathrm{PMA})_{2} \mathrm{PbI}_{4}$ structures. It is clear that the displacement of I atom, whose orbitals form the valence band maximum (VBM) and conduction band minimum $(\mathrm{CBM})$, is suppressed in $(\mathrm{PVBA})_{2} \mathrm{PbI}_{4}$. The displacement of heavy $\mathrm{Pb}$ atoms is much smaller than the I atoms. The atom displacement cause fluctuations in the electronic bandstructure. Obviously, the time variation of both $\mathrm{VBM}$ and $\mathrm{CBM}$ in (PVBA) $)_{2} \mathrm{PbI}_{4}$ are smaller than that of (PMA) ${ }_{2} \mathrm{PbI}_{4}$ structure (Fig. $3 c)$.

The higher rigidity results in less activated optical phonon modes and weaker EPC in $(\mathrm{PVBA})_{2} \mathrm{PbI}_{4}$ perovskite ${ }^{14}$. Temperature-dependent steady-state $\mathrm{PL}$ was conducted to investigate the strength of EPC in the perovskite films (Supplementary Fig. 7). It has been 
demonstrated that EPC dominates the line broadening in various $2 \mathrm{D}$ perovskite, and the contribution from impurities can be neglected ${ }^{31}$. As shown in Fig. 3d,e, the $\left(\mathrm{PVBA}_{2} \mathrm{PbI}_{4}\right.$ film has obviously smaller full-width at half-maximum (FWHM) than the control (PMA) ${ }_{2} \mathrm{PbI}_{4}$ film, particularly at high temperature.. The deformation potential $(D)$ defined as the shift in energy band per unit strain ${ }^{14}$, which can be used to quantify the EPC strength, is reduced from $1.3 \times 10^{9}$ $\mathrm{eV} / \mathrm{cm}$ in (PMA) $)_{2} \mathrm{PbI}_{4}$ to $8.7 \times 10^{8} \mathrm{eV} / \mathrm{cm}$ in (PVBA) ${ }_{2} \mathrm{PbI}_{4}$ (see Methods). perovskite $^{32}$. Both temperature-dependent PLQY and carrier lifetime $\left(T_{\text {average }}\right)$ were measured to examine the recombination dynamics of the polymerized perovskite (Supplementary Fig. 7). Radiative and non-radiative recombination rate ( $k_{\text {rad }}$ and $k_{n o n}$, respectively) are calculated using equations $\frac{1}{T_{\text {average }}}=k_{\text {rad }}+k_{\text {non }}$ and PLQY $=\frac{k_{\text {rad }}}{k_{\text {rad }}+k_{\text {non }}}$. As shown in Fig. 3f,g, the $k_{\text {non }}$ of $(\mathrm{PMA})_{2} \mathrm{PbI}_{4}$ increase dramatically with temperature up to $380 \mathrm{~K}$, in contrast to the slight increase of $(\mathrm{PVBA})_{2} \mathrm{PbI}_{4}$ films. Considering that the trap densities of $(\mathrm{PVBA})_{2} \mathrm{PbI}_{4}$ and $(\mathrm{PMA})_{2} \mathrm{PbI}_{4}$ calculated by space-charge limited current (SCLC) measurement are similar therefore result from the EPC difference (Fig. 3f,g).

In addition to charge recombination dynamics, EPC also influence charge transportation due to phonon scattering ${ }^{33,34}$. Temperature-dependent SCLC was measured to examine the influence of EPC on carrier mobility (Supplementary Fig. 9). As shown in Fig. 3h,i, two regimes are identified corresponding to orthorhombic and tetragonal phases. Both regimes can be fitted by a power law, $\mu \propto T^{-m}$, where $\mathrm{m}$ is a co-efficient indicating strength of $\mathrm{EPC}^{35}$. In 
both regimes, the exponent (m) of (PVBA $)_{2} \mathrm{PbI}_{4}$ are smaller than that of $(\mathrm{PMA})_{2} \mathrm{PbI}_{4}$ for both electron and hole mobility, indicating that weaker EPC in the polymerized perovskite.

\section{The polymerized PeLEDs.}

As an application of polymerized perovskite, we fabricated PeLEDs using (PVBA) ${ }_{2} \mathrm{MA}_{\mathrm{n}}$ ${ }_{1} \mathrm{~Pb}_{n} \mathrm{I}_{3 \mathrm{n}+1}$ films with a structure of ITO/poly-TPD/perovskite/TPBi/LiF/Al (ITO: indium tin oxide, poly-TPD: poly[N,N'-bis (4-butylphenyl)-N,N'-bis(phenyl)-benzidine], TPBi: 2,2',2"(1,3,5-Benzinetriyl)-tris(1- phenyl-1-H-benzimidazole)). It should be noted that the quasi-2D perovskite films consist several phases with different $n$ values. Fig. 4a shows energy level diagram of the devices, and the carriers transfer from large bandgap perovskite (small $\mathrm{n}$ phases) to small bandgap ones (large $n$ phases) ${ }^{36,37}$. The strong EPC in small $n$ phases causes nonradiative recombination and thus charge loss during the energy transfer process (Fig. 4a). The polymerized perovskite with weak EPC suppresses this loss and results in higher PLQY. For example, the PLQY increases from $32.8 \%$ for control (PMA) $)_{2}(\mathrm{MA})_{9} \mathrm{~Pb}_{10} \mathrm{I}_{31}$ film to $45.7 \%$ for (PVBA) $2(\mathrm{MA})_{9} \mathrm{~Pb}_{10} \mathrm{I}_{31}$ film, which should be attributed to their different EPC as both films have similar trap density (Supplementary Fig. 8). The EQE of the PeLEDs increase from 12.5\% to a decent value of $17.6 \%$ accordingly (Fig. $4 \mathrm{~b}$ ). The EQEs of polymerized perovskite $(\mathrm{PVBA})_{2} \mathrm{MA}_{\mathrm{n}-1} \mathrm{~Pb}_{\mathrm{n}} \mathrm{I}_{3 \mathrm{n}+1}(\mathrm{n}=2,5,7,10,20)$ based PeLEDs are much higher than that of control PeLEDs, particularly for devices with small $<\mathrm{n}>$ films $(<\mathrm{n}>$ is the value for stoichiometric quasi-2D perovskite in solution) (Fig. 4c). The EQE is further improved to a record value of $23.2 \%$, approaching to the out-coupling limit of $24.7 \%$ (Supplementary Fig. 10), using popular mixed-cations and mixed-halides perovskite $(\mathrm{PVBA})_{2}\left(\mathrm{FA}_{0.83} \mathrm{Cs}_{0.17}\right)_{9} \mathrm{~Pb}_{10}\left(\mathrm{I}_{0.9} \mathrm{Br}_{0.1}\right)_{31} \quad(\mathrm{FA}$ : 
cation perovskites $^{38}$, evidenced by its higher external PLQY value of $56.8 \%$. The internal

224

225

226

227

228

229

230

231

232

233

234

235

236

237

238

239

240

241

242

243

244

quantum yield reaches $93.5 \%$ assuming both charge carrier balance and radiative excitons are unity. This value is slightly different with that calculated from the PLQY values $(91.2 \%$, as the escape probability of photon is $12.7 \%^{39}$ ) due to their different carrier concentrations. The current density-voltage $(J-V)$, angular spectra and intensity profiles, and EQE curves of the devices are shown in Supplementary Fig. 11-12. Notably, the electroluminescence (EL) spectra of polymerized perovskite based PeLEDs are generally sharper than that of (PVBA) ${ }_{2} \mathrm{MA}_{\mathrm{n}}$ ${ }_{1} \mathrm{~Pb}_{\mathrm{n}} \mathrm{I}_{3 \mathrm{n}+1}$ PeLEDs (Fig. 4d), further demonstrating weaker EPC in the emissive low-bandgap domains in the PeLEDs.

We conducted temperature-dependent measurement to examine the effect of EPC on device performance using $<\mathrm{n}>=2$ perovskites as examples because there are more small $\mathrm{n}$ phases in the films (Supplementary Fig. 13). As shown in Fig. 4e, the EQE of the control PeLEDs decrease dramatically with temperature compared to that of (PVBA) ${ }_{2} \mathrm{MAPb}_{2} \mathrm{I}_{7}$ based PeLEDs. This result demonstrates that the EPC plays a critical role in PeLEDs.

We finally measured the operation stability ( $\mathrm{T}_{50}$, time to half of the initial luminance) under a constant current density of $5 \mathrm{~mA} / \mathrm{cm}^{2}$ in a $\mathrm{N}_{2}$ glove-box without encapsulation. The $\mathrm{T}_{50}$ of (PVBA) ${ }_{2} \mathrm{MAPb}_{2} \mathrm{I}_{7}$ based PeLEDs reached $20.8 \mathrm{~h}$, much longer than the control PeLEDs (Fig. 4f). The better operation stability can be ascribed to the more stable structure of polymerized perovskite than (PMA) ${ }_{2} \mathrm{MAPb}_{2} \mathrm{I}_{7}$.

\section{Discussion}

We have demonstrated the formation of polymerized perovskite using unsaturated organoammonium ligands. The polymerized perovskites show polymer-like behavior such as 
improved film stability and flexibility, in contrast to regular RP and DJ phases with ammonium functional monomers. Compared to various polymer additives reported previously

247 (Supplementary Table 1), a unique advantage for polymerized perovskite is that PVBA ligands are in the perovskite lattice and forms perovskite structure with metal-halide octahedron. Benefitted from the polymerized perovskite structure, the strong $\mathrm{C}-\mathrm{C}$ covalent bond in PVBA can reduce ion vibration of metal-halide octahedron and increase lattice rigidity. The increased

251 lattice rigidity further suppresses EPC of OHPs, leading to suppressed nonradiative recombination rate, small PL/EL FWHM, as well as increased carrier mobility without sacrificing its tolerance to defects. PeLEDs made from $(\mathrm{PVBA})_{2}\left(\mathrm{FA}_{0.83} \mathrm{Cs}_{0.17}\right)_{9} \mathrm{~Pb}_{10} \mathrm{I}_{31}$ showed a decent EQE of $23.2 \%$ with improved operation stability. Our discovery of polymer perovskite opens up a new way to design perovskites to improve their intrinsic stability, and tune the physical property of perovskites.

Methods

Materials. Chemicals used in the experiments include lead iodide ( $\mathrm{PbI}_{2} ; 99.999 \%$, Alfa-Aesar), cesium iodide (CsI; 99.999\%, Alfa-Aesar), formamidinium iodide (FAI; 99\%, Xi'an Polymer Light Technology Corp), lead bromide $\left(\mathrm{PbBr}_{2} ; 99.999 \%\right.$, Alfa-Aesar), poly[N,N'-bis (4butylphenyl)-N,N'-bis(phenyl)-benzidine] (poly-TPD; American Dye Source Inc.), 2,2',2"(1,3,5-Benzinetriyl)-tris(1-phenyl-1-H-benzimidazole) (TPBi; Lumtech Inc.), lithium fluoride (LiF; 98.5\%; Alfa-Aesar), N,N-dimethylformamide (DMF; 99.8\%, Sigma-Aldrich), chlorobenzene (CB; 99.8\%, Sigma-Aldrich), toluene (99.8\%, Sigma-Aldrich). 
$99 \%$, Sigma-Aldrich) with equimolar amounts of aqueous hydriodic acid (HI; 57 wt. $\%$ in $\mathrm{H}_{2} \mathrm{O}$,

268

269

270

271

272

273

Sigma-Aldrich) at $0{ }^{\circ} \mathrm{C}$ with constant stirring for $2 \mathrm{~h}$. The solvent was then evaporated under reduced pressure at $65{ }^{\circ} \mathrm{C}$. The remaining white solid product were purified by recrystallization in ethanol, and the recrystallized solid were dried in the $\mathrm{N}_{2}$ glove-box at $60{ }^{\circ} \mathrm{C}$ for $24 \mathrm{~h}$. VBAI was synthesized using a similar procedure with PMAI. 4-vinybenzylamine (92\%, stabilized with 4-methoxyphenol, TCI) was firstly disolved in cold ethanol (cooled in an ice bath), and then mixed with equal molar amount of $\mathrm{HI}$ solution and stirred at $0{ }^{\circ} \mathrm{C}$ for $2 \mathrm{~h}$. VBAI solids were obtained by adding diethyl ether in the mixed solution, followed by filtration and wash using diethyl ether. The VBAI solids was finally dried in the $\mathrm{N}_{2}$ glove-box at $50{ }^{\circ} \mathrm{C}$ for $24 \mathrm{~h}$.

Peroskite film preparation and characterizations. Quasi-2D perovskite (VBA $)_{2}(\mathrm{MA})_{\mathrm{n}-1} \mathrm{~Pb}_{\mathrm{n}} \mathrm{I}_{3 \mathrm{n}+1}$ (or (PMA) $2(\mathrm{MA})_{\mathrm{n}-1} \mathrm{~Pb}_{\mathrm{n}} \mathrm{I}_{3 \mathrm{n}+1}$ ) solution was prepared by dissolving stoichiometric quantities of $\mathrm{PbI}_{2}, \mathrm{MAI}$ and VBAI (or PMAI) mixture in DMF with a concentration of $0.3 \mathrm{M}$, and stirred for overnight before use. Quasi-2D perovskite $(\mathrm{VBA})_{2}\left(\mathrm{FA}_{0.83} \mathrm{Cs}_{0.17}\right)_{\mathrm{n}-1} \mathrm{~Pb}_{\mathrm{n}}\left(\mathrm{I}_{0.9} \mathrm{Br}_{0.1}\right)_{3 \mathrm{n}+1}$ (or $\left.(\mathrm{PMA})_{2}\left(\mathrm{FA}_{0.83} \mathrm{Cs}_{0.17}\right)_{\mathrm{n}-1} \mathrm{~Pb}_{\mathrm{n}}\left(\mathrm{I}_{0.9} \mathrm{Br}_{0.1}\right)_{3 \mathrm{n}+1}\right)$ solution was prepared using a similar method. The perovskite solutions were spin coated on cleaned glass substrates at 5,000 r.p.m. for $60 \mathrm{~s}$, and toluene was dropped on the spinning substrate at around 1-2 seconds before the spinning the perovskite film change color. The thickness of perovskite films, around $50 \mathrm{~nm}$, was measured using a Dektak XT profiler. The perovskite films were annealed at $80{ }^{\circ} \mathrm{C}$ for $10 \mathrm{~min}$ to remove solvent residue. The polymerization process was conducted using a UV lamp (wavelength: 254 $\mathrm{nm}$; intensity: $\left.0.1 \mathrm{~W} / \mathrm{cm}^{2}\right)$.

XRD measurements were performed with a SmartLab diffractometer with the BraggBrentano parallel beam geometry, and a conventional $\mathrm{Cu}$ target $\mathrm{X}$-ray tube was set to $40 \mathrm{kV}$ 
and $150 \mathrm{~mA}$. FTIR measurements were conducted using the Nicolet 8700 with a spectral range from 4000 to $500 \mathrm{~cm}^{-1}$, with an Attenuated Total Reflection (ATR) accessory. ${ }^{1} \mathrm{H}$ NMR spectra were acquired at room temperature on Bruker AVANCE III NMR Spectormeters with a 400 MHz Bruker magnet. All chemical shifts were reported in ppm relative to tetramethylsilane (TMS). Films were redissolved in $\mathrm{d}_{6}$-DMSO for measurements. GPC were measured with Waters PL-GPC50 apparatus, and DMF were employed as an eluent at a flow rate of 1.0 $\mathrm{mL} / \mathrm{min}$. Films were redissolved in DMF for measurements. The SEM images were collected in a GeminiSEM 500 with an accelerating voltage of $3 \mathrm{kV}$. The HRTEM images were obtained using a JEM-2100F system. TGA measurements were acquired via a STA 6000 thermal analysis system, heating at a rate of $10{ }^{\circ} \mathrm{C} / \mathrm{min}$ from 30 to $700{ }^{\circ} \mathrm{C}$ under a constant $50 \mathrm{~mL} / \mathrm{min}$ $\mathrm{N}_{2}$ gas flow. UPS data were obtained using a ESCALAB 250Xi spectrometer with He I radiation $(21.2 \mathrm{eV})$ at a pass energy $(\mathrm{PE})$ of $2 \mathrm{eV}$. Absorption spectra were measured using a Perkin Elmer Lambda 950 UV-vis-NIR spectrometer. The steady-state PL and PLQY data were measured using a Horiba Fluorolog-3 system with Petite Integrating Sphere. The excitation wavelength was $450 \mathrm{~nm}$ provided by a Xe lamp. The TRPL were taken using a Horiba timecorrelated single-photon counting system. The samples were excited by a pulsed laser diode (NanoLED-Horiba) with a center wavelength of $369 \mathrm{~nm}$, an excitation intensity of $0.6 \mathrm{~mW} / \mathrm{cm}^{2}$, and a repetition rate that was less than the reciprocal of the measurement range. The TRPL curves were fitting with 2-exponential components. The refractive index of perovskite films was measured by a J. A. Woollam variable angle spectroscopic ellipsometer. All samples were spin-coated on Si wafers and the spectra were ftted using the model of "Si with absorbing film." 
311 (Optistat DN-V, Oxford Instruments). Temperature was controlled by a cryogenic environment 312 controller (Mercury iTC, Oxford Instruments). All temperatures were stabilized for $15 \mathrm{~min}$ 313 before measurement. The PLQY values at different temperatures were calibrated using PLQY 314 values at room temperature.

315 PeLED fabrication and characterizations. Pre-patterned ITO substrates were sequentially sonicated with deionized water, acetone, and ethanol for $20 \mathrm{~min}$. The substrates were treated by ultraviolet-ozone for $10 \mathrm{~min}$ before use. Poly-TPD solution $(6 \mathrm{mg} / \mathrm{mL}$ in $\mathrm{CB})$ was spincoated on the ITO substrates at $1,000 \mathrm{rpm}$ for $60 \mathrm{~s}$, followed by thermal annealing at $150{ }^{\circ} \mathrm{C}$ for $20 \mathrm{~min}$. The poly-TPD layer was treated by $\mathrm{O}_{2}$ plasma for $5 \mathrm{~s}$ to improve wetting. TPBi, LiF and Al layers were sequentially evaporated on top of the perovskite film with thicknesses of 40, 1.2 and $100 \mathrm{~nm}$, respectively. The device area was $0.04 \mathrm{~cm}^{2}$.

The performance of PeLEDs were measured in a $\mathrm{N}_{2}$ glove-box using a homemade motorized goniometer setup consisting of a Keithley 2400 sourcemeter unit, a calibrated Si photodiode (FDS-100-CAL, Thorlabs), a picoammeter (4140B, Agilent), and a calibrated fiber optic spectrophotometer (UVN-SR, StellarNet Inc.). The current density-voltage (J-V) curve was obtained by scanning the voltage from -0.5 to $5 \mathrm{~V}$ (forward scan) with a step of $0.05 \mathrm{~V}$. Temperature-dependent of PeLED and SCLC measurement were conducted using $\mathrm{N}_{2}$ cryostat mentioned above. Si photodiode and fiber probe were attached to the quartz window of the cryostat. The EQEs values at different temperatures were calibrated using EQE value at room temperature.

Computational method. Ab initio molecular dynamics (AIMD) simulations and electronic structure calculations were performed using the Vienna ab initio simulation package (VASP) ${ }^{40}$. 
Projector-augmented wave pseudopotentials were employed to describe the electron-nuclei interactions $^{41,42}$, and the Perdew-Burke-Ernzerhof (PBE) exchange-correlation functional were used in all calculations ${ }^{43}$. The Kohn-Sham wave functions were expanded in plane waves up to $400 \mathrm{eV}$. We used a $3 \times 3 \times 1$ mesh grid to sample the K-space uniformly. The convergence threshold of energy in the self-consistent step was set to $10^{-5} \mathrm{eV}$ with Gaussian smearing of $0.01 \mathrm{eV} / \AA$. Moreover, the force threshold of geometry optimation is $0.02 \mathrm{eV} / \AA$ for each dimension of all atoms in the supercell. The elastic tensor was determined by performing six finite lattice distortions, and the elastic constants were derived from the strain-stress 341 relationship ${ }^{44}$. The effective masses of free carriers were derived by fitting E-k diagram near band extremum by a parabola. Ion-clamped dielectric constants of anionic and organic layers were estimated in frozen configurations using density functional perturbation theory ${ }^{45}$. AIMD were used to reveal atomic displacement at specific temperature $(80,300$ and $380 \mathrm{~K}$ after geometry optimization). Then, a 5 ps microcanonical AIMD trajectory was then generated with

a time step of 1 fs. Finally, the root mean squared displacement (RMSD) was evaluated by comparing to optimum configuration. The phonons at $\Gamma$ point were calculated by finitedisplacement method implemented in the PHONOPY package with step of $0.015 \AA^{46}$. Here,we taken VASP as the force-constant calculator with a high plane waves cutoff at $500 \mathrm{eV}$, and the force evaluations were performed on $1 \times 1 \times 1$ supercells using k-point sampling meshes $2 \times 2 \times 2$.

Deformation potential calculation. Deformation potential $(D)$ is defined as the change in the bandgap energy per unit strain due to phonon scattering, which has been used to describe the electron-phonon interation ${ }^{47}$. To estimate $D$, the temperature-dependent FWHM of 


$$
\Gamma(T)=\Gamma_{0}+\gamma_{a c} T+\frac{\Gamma_{L O}}{e^{\hbar \omega_{L O} / k_{B} T}-1}+\Gamma_{i m p} e^{-E_{i m p} / k_{B} T}
$$

where $\Gamma_{0}$ is the temperature-independent inhomogeneous broadening; $\gamma_{a c}$ is the acoustic phonon interaction constant; $\Gamma_{L O}$ is the optical phonon interaction constant; $\hbar \omega_{L O}$ represents the optical phonon energy; $\Gamma_{\text {imp }}$ is the PL broadening from the completely ionized impurity states; $E_{\text {imp }}$ is the average energy of the impurities ionizing, and $k_{B}$ and $T$ correspond to the Boltzmann constant and the temperature, respectively.

The homopolar optical phonon scattering has been proved to be the main sources of scattering by the relaxation mechanism at high temperature ${ }^{14}$. Thus, the contribution of charge impurities and acoustic phonons to the broadening can be ignaored ${ }^{16}$ :

$$
F W H M=\Gamma_{0}+\frac{\Gamma_{h o m o}}{e^{\hbar \omega_{h o m o} / k_{B} T}-1}
$$

At high temperature, $\Gamma_{\text {homo }}$ is approximated as:

$$
\Gamma_{\text {homo }}=\frac{\hbar M \omega_{\text {homo }}}{\rho L}\left(\frac{D}{\hbar \omega_{\text {homo }}}\right)^{2}
$$

where $M=m_{e}{ }^{*}+m_{h}{ }^{*}$ is the sum of electron and hole effective masses (Supplementary Table 2); Whomo is the homopolar phonon angular frequency; $\rho$ is the mass density $\left(2.7 \mathrm{~g} / \mathrm{cm}^{3}\right.$ for both materials $)^{16} ; L$ is the thickness of quantum well where the excition is confined $(L=0.64 \mathrm{~nm}$ for $\mathrm{Pb}-\mathrm{I}-\mathrm{Pb}$ inorganic layer).

Trap density and carrier mobility caclulation. The trap density and carrier mobility were caclulated using SCLC results. The hole-only device structure is ITO/poly-TPD $(40 \mathrm{~nm}) / 2 \mathrm{D}$ perovskite $(100 \mathrm{~nm}) /$ poly-TPD $(40 \mathrm{~nm}) / \mathrm{MoO}_{3}(10 \mathrm{~nm}) / \mathrm{Ag}(100 \mathrm{~nm})$ (ITO: indium tin oxide, poly-TPD: poly[N,N'-bis (4-butylphenyl)-N,N'-bis(phenyl)-benzidine]). The electron-only device structure is $\mathrm{ITO} / \mathrm{SnO}_{2}(20 \mathrm{~nm}) / 2 \mathrm{D}$ perovskite $(100 \mathrm{~nm}) / \mathrm{TPBi}(40 \mathrm{~nm}) / \mathrm{LiF}(1.2 \mathrm{~nm}) / \mathrm{Al}$

$376 \quad(100 \mathrm{~nm}) \quad$ (TPBi: 2,2',2"-(1,3,5-Benzinetriyl)-tris(1-phenyl-1-H-benzimidazole)). Three 
distinct regions could be identified from the $I-V$ curves including Ohmic, trap-filled limited (TFL) as well as SCLC regions. The hole and electron trap density were extract from hole and electron only devices, respectively, using equation ${ }^{49}$

$$
N_{\text {traps }}=2 \varepsilon_{0} \varepsilon_{r} V_{T F L} /\left(q L^{2}\right)
$$

where the $\varepsilon_{0}, \varepsilon_{r}, V_{T F L}, q$ and $L$ are the vacuum permittivity, the relative dielectric constant, the onset voltage of the TFL region, elementary charge and the thickness of the perovskite films, respectively.

The charge carrier mobility was extracted from the SCLC regions using the Mott-Gurney $\operatorname{Law}^{49}$ :

$$
J=\frac{9 \varepsilon_{0} \varepsilon_{r} \mu V^{2}}{8 L^{3}}
$$

where $\mu$ is the charge mobility, $J$ and $V$ are current density and applied voltage, respectively.

Optical modelling. The light outcoupling calculations were based on a classical dipole emission model $^{50}$. The power ratio radiated to each in-plane wavevector range (outcoupled, substrate, waveguided, surface plasmon polariton) was simulated by using the experimentally obtained optical constants (refractive index) of each layer to construct the model device. The refractive indexes of perovskite were obtained by ellipsometry. The dipole position was assumed to be at the center of perovskite layer.

\section{Data availability}

The data that support the findings of this study are available from the corresponding author upon reasonable request.

\section{References}

1. Liu, X. K. et al. Metal halide perovskites for light-emitting diodes. Nat. Mater. 20, 10-21 
400

401

402

403

404

405

406

407

408

409

410

411

412

413

414

415

416

417

418

419

420

2. Kim, G. et al. Impact of strain relaxation on performance of alpha-formamidinium lead iodide perovskite solar cells. Science 370, 108-112 (2020).

3. Wei, H. \& Huang, J. Halide lead perovskites for ionizing radiation detection. Nat. Commun. 10, 1066 (2019).

4. Nayak, P. K., Mahesh, S., Snaith, H. J. \& Cahen, D. Photovoltaic solar cell technologies: analysing the state of the art. Nat. Rev. Mater. 4, 269-285 (2019).

5. Thouin, F. et al. Phonon coherences reveal the polaronic character of excitons in twodimensional lead halide perovskites. Nat. Mater. 18, 349-356 (2019).

6. Chu, W., Zheng, Q., Prezhdo, O. V., Zhao, J. \& Saidi, W. A. Low-frequency lattice phonons in halide perovskites explain high defect tolerance toward electron-hole recombination. Sci. $A d v$. 6, eaaw7453 (2020).

7. Shi, E. et al. Two-dimensional halide perovskite lateral epitaxial heterostructures. Nature 580, 614-620 (2020).

8. Akkerman, Q. A., Raino, G., Kovalenko, M. V. \& Manna, L. Genesis, challenges and opportunities for colloidal lead halide perovskite nanocrystals. Nat. Mater. 17, 394-405 (2018).

9. Wang, Y. et al. Stabilizing heterostructures of soft perovskite semiconductors. Science 365, 687-691 (2019).

10. Wright, A. D. et al. Electron-phonon coupling in hybrid lead halide perovskites. Nat. Commun. 7, 11755 (2016).

11. Cho, H. et al. High-Efficiency Solution-Processed Inorganic Metal Halide Perovskite 
422

423

424

425

426

427

428

429

430

431

432

433

434

435

436

437

438

439

440

441

442

12. Ren, H. et al. Efficient and stable Ruddlesden-Popper perovskite solar cell with tailored interlayer molecular interaction. Nat. Photonics 14, 154-163 (2020).

13. Chen, Z., Guo, Y., Wertz, E. \& Shi, J. Merits and Challenges of Ruddlesden-Popper Soft Halide Perovskites in Electro-Optics and Optoelectronics. Adv. Mater. 31, 1803514 (2019).

14. Gong, X. et al. Electron-phonon interaction in efficient perovskite blue emitters. Nat. Mater. 17, 550-556 (2018).

15. Zhang, L. et al. Bright Free Exciton Electroluminescence from Mn-Doped TwoDimensional Layered Perovskites. J. Phys. Chem. Lett. 10, 3171-3175 (2019).

16. Guo, Z., Wu, X., Zhu, T., Zhu, X. \& Huang, L. Electron-Phonon Scattering in Atomically Thin 2D Perovskites. ACS Nano 10, $9992-9998$ (2016).

17. Era, M., Morimoto, S., Tsutsui, T. \& Saito, S. Organic - inorganic heterostructure electroluminescent device using a layered perovskite semiconductor $\left(\mathrm{C}_{6} \mathrm{H}_{5} \mathrm{C}_{2} \mathrm{H}_{4} \mathrm{NH}_{3}\right)_{2} \mathrm{PbI}_{4}$. Appl. Phys. Lett. 65, 676-678 (1994).

18. Li, X. et al. In-situ cross-linking strategy for efficient and operationally stable methylammoniun lead iodide solar cells. Nat. Commun. 9, 3806 (2018).

19. Wang, R. et al. Constructive molecular configurations for surface-defect passivation of perovskite photovoltaics. Science 366, 1509-1513 (2019).

20. Na Quan, L. et al. Edge stabilization in reduced-dimensional perovskites. Nat. Commun. 11, 170 (2020).

21. Zhao, Y. et al. A Polymerization-Assisted Grain Growth Strategy for Efficient and Stable Perovskite Solar Cells. Adv. Mater. 32, e1907769 (2020). 
22. Guo, P. et al. Double Barriers for Moisture Degradation: Assembly of Hydrolysable Hydrophobic Molecules for Stable Perovskite Solar Cells with High Open-Circuit Voltage. Adv. Funct. Mater. 30, 2002639 (2020).

23. Bi, D. et al. Polymer-templated nucleation and crystal growth of perovskite films for solar cells with efficiency greater than 21\%. Nat. Energy 1, 16142 (2016).

24. Tieke, B. \& Chapuis, G. Solid State Polymerization of Butadienes in Layer Structures. Mol. Cryst. Liq. Cryst. 137, 101-116 (1986).

25. Kosuge, H., Okada, S., Oikawa, H. \& Nakanishi, H. Polydiacetylenes in Organic-Inorganic Hybrid Systems. Mol. Cryst. Liq. Cryst. 377, 13-18 (2002).

26. Proppe, A. H. et al. Photochemically Cross-Linked Quantum Well Ligands for 2D/3D Perovskite Photovoltaics with Improved Photovoltage and Stability. J. Am. Chem. Soc. 141, 14180-14189 (2019).

27. Deng, J.-P., Yang, W.-T. \& Rånby, B. Auto-Initiating Performance of Styrene on Surface Photografting Polymerization. Macromol. Rapid Commun. 22, 535-538 (2001).

28. Tremblay, M.-H. et al. Structures of $\left(4-\mathrm{Y}_{-} \mathrm{C}_{6} \mathrm{H}_{4} \mathrm{CH}_{2} \mathrm{NH}_{3}\right)_{2} \mathrm{PbI}_{4}\{\mathrm{Y}=\mathrm{H}, \mathrm{F}, \mathrm{Cl}, \mathrm{Br}, \mathrm{I}\}$ : Tuning of Hybrid Organic Inorganic Perovskite Structures from Ruddlesden-Popper to DionJacobson Limits. Chem. Mater. 31, 6145-6153 (2019).

29. Mehdizadeh, A., Akhtarianfar, S. F. \& Shojaei, S. Role of Methylammonium Rotation in Hybrid Halide $\mathrm{MAPbX}_{3}(\mathrm{X}=\mathrm{I}, \mathrm{Br}$, and $\mathrm{Cl})$ Perovskites by a Density Functional Theory Approach: Optical and Electronic Properties. J. Phys. Chem. C 123, 6725-6734 (2019).

30. Gonzalez-Carrero, S., Espallargas, G. M., Galian, R. E. \& Pérez-Prieto, J. Blueluminescent organic lead bromide perovskites: highly dispersible and photostable 
materials. J. Mater. Chem. A 3, 14039-14045 (2015).

466

467

468

469

470

471

472

473

474

475

476

477

478

479

480

481

482

483

484

485

486

31. Ni, L. et al. Real-Time Observation of Exciton-Phonon Coupling Dynamics in SelfAssembled Hybrid Perovskite Quantum Wells. ACS Nano 11, 10834-10843 (2017).

32. Chu, W., Saidi, W. A., Zhao, J. \& Prezhdo, O. V. Soft Lattice and Defect Covalency Rationalize Tolerance of $\beta-\mathrm{CsPbI}_{3}$ Perovskite Solar Cells to Native Defects. Angew. Chem. Int. Ed. 59, 6435-6441 (2020).

33. Herz, L. M. Charge-Carrier Mobilities in Metal Halide Perovskites: Fundamental Mechanisms and Limits. ACS Energy Lett. 2, 1539-1548 (2017).

34. Karakus, M. et al. Phonon-Electron Scattering Limits Free Charge Mobility in Methylammonium Lead Iodide Perovskites. J. Phys. Chem. Lett. 6, 4991-4996 (2015).

35. Shrestha, S. et al. Assessing Temperature Dependence of Drift Mobility in Methylammonium Lead Iodide Perovskite Single Crystals. J. Phys. Chem. C 122, 59355939 (2018).

36. Yuan, M. et al. Perovskite energy funnels for efficient light-emitting diodes. Nat. Nanotech. 11, 872-877 (2016).

37. Wang, N. et al. Perovskite light-emitting diodes based on solution-processed selforganized multiple quantum wells. Nat. Photonics 10, 699-704 (2016).

38. Rehman, W. et al. Photovoltaic mixed-cation lead mixed-halide perovskites: links between crystallinity, photo-stability and electronic properties. Energy Environ. Sci. 10, 361-369 (2017).

39. Richter, J. M. et al. Enhancing photoluminescence yields in lead halide perovskites by photon recycling and light out-coupling. Nat. Commun. 7, 13941 (2016). 
487

488

489

490

491

492

493

494

495

496

497

498

499

500

501

502

503

504

505

506

507

508

40. Kresse, G. \& Hafner, J. Ab initio molecular dynamics for open-shell transition metals. Phys. Rev. B 48, 13115-13118 (1993).

41. Blochl, P. E. Projector augmented-wave method. Phys. Rev. B 50, 17953-17979 (1994).

42. Kresse, G. \& Joubert, D. From ultrasoft pseudopotentials to the projector augmented-wave method. Phys. Rev. B 59, 1758-1775 (1999).

43. Perdew, J. P., Burke, K. \& Ernzerhof, M. Generalized Gradient Approximation Made Simple. Phys. Rev. Lett. 77, 3865-3868 (1996).

44. Le Page, Y. \& Saxe, P. Symmetry-general least-squares extraction of elastic data for strained materials fromab initiocalculations of stress. Phys. Rev. B 65, 104104 (2002).

45. Gajdoš, M., Hummer, K., Kresse, G., Furthmüller, J. \& Bechstedt, F. Linear optical properties in the projector-augmented wave methodology. Phys. Rev. B 73, 045112 (2006).

46. Chaput, L., Togo, A., Tanaka, I. \& Hug, G. Phonon-phonon interactions in transition metals. Phys. Rev. B 84, 094302 (2011).

47. Franceschetti, A., Wei, S. H. \& Zunger, A. Absolute deformation potentials of Al, Si, and NaCl. Phys. Rev. B 50, 17797-17801 (1994).

48. Handa, T., Aharen, T., Wakamiya, A. \& Kanemitsu, Y. Radiative recombination and electron-phonon coupling in lead-free $\mathrm{CH}_{3} \mathrm{NH}_{3} \mathrm{SnI}_{3}$ perovskite thin films. Phys. Rev. Mater. 2, 075402 (2018)

49. Dong, Q. et al. Electron-hole diffusion lengths $>175 \mu \mathrm{m}$ in solution-grown $\mathrm{CH}_{3} \mathrm{NH}_{3} \mathrm{PbI}_{3}$ single crystals. Science 347, 967-970 (2015).

50. Furno, M., Meerheim, R., Hofmann, S., Lüssem, B. \& Leo, K. Efficiency and rate of spontaneous emission in organic electroluminescent devices. Phys. Rev. B 85, 115205 


\section{Acknowledgements}

511 We acknowledge the support from the National Natural Science Foundation of China (NSFC,

512 Award \# 51872274) and the Fundamental Research Funds for the Central Universities

513 (WK2060190053). This work was partially carried out at the University of Science and

514 Technology of China Center for Micro and Nanoscale Research and Fabrication.

\section{Author contributions}

516 Z.X. conceived the idea and supervised the project. W.C. fabricated and characterized the 517 perovskite films and PeLEDs. Y.S., Y.L. and J.Z. did the theoretical simulation. J.C. carried out 518 the temperature-dependent SCLC measurement. P.M. did the optical simulations of the 519 PeLEDs. Z.F. conducted the absorption and PL characterizations. Y.D. and W.C. synthesized 520 VBAI. Z.X. and W.C. wrote the manuscript, and all authors reviewed the manuscript.

\section{Competing financial interests}

522 The authors declare no competing financial interests. 


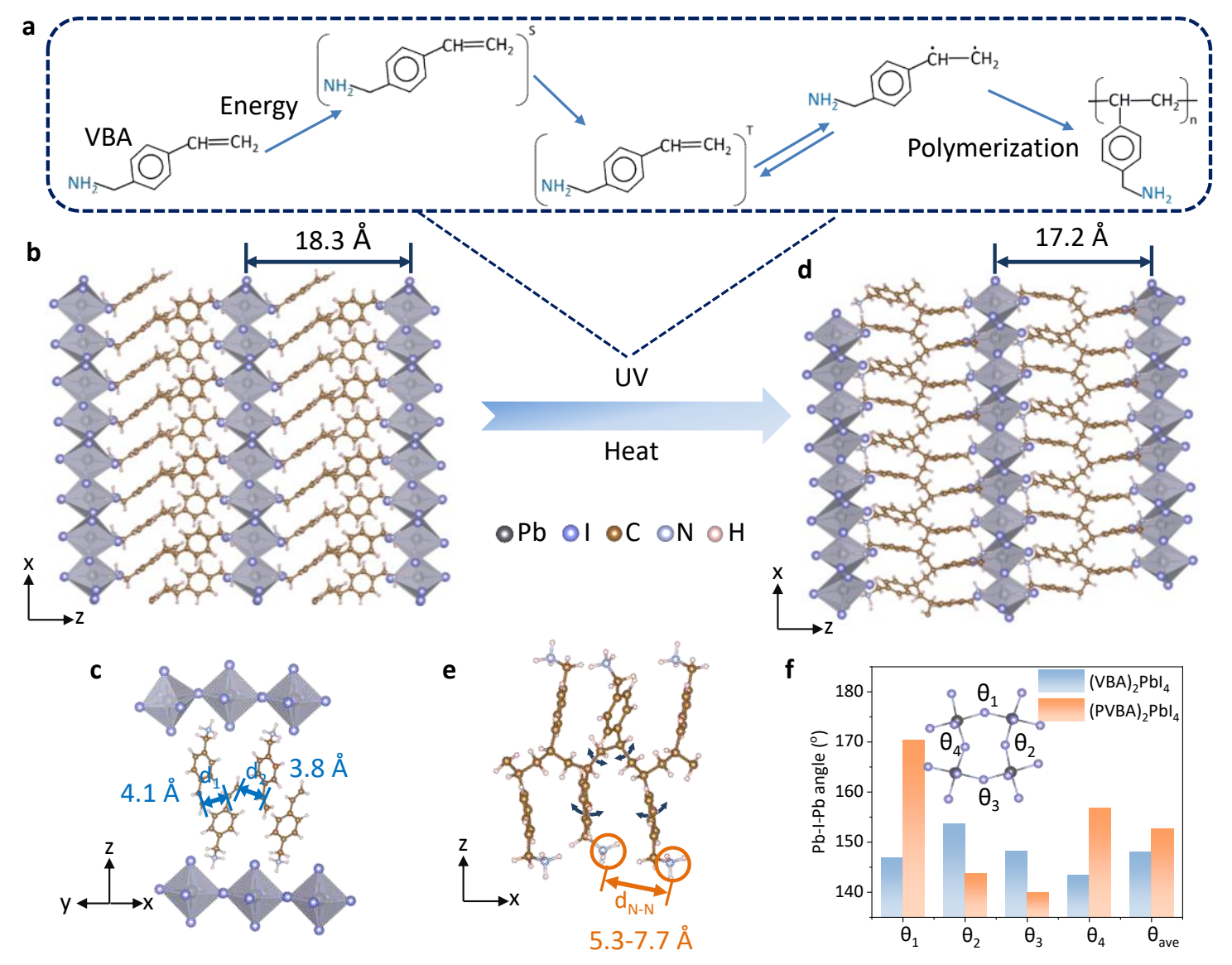

Fig. 1 | Theoretical calculation of polymerized perovskite structure. a, The polymerization

527 process of VBA monomers. b,c, Molecular structure of $(\mathrm{VBA})_{2} \mathrm{PbI}_{4}$ perovskite (b) and the

528 VBA monomer configuration in the perovskite lattice (c). $d_{1}$ and $d_{2}$ represent the distance between two adjacent $\mathrm{C}=\mathrm{C}$ functional groups in the perovskite lattice. d,e, Molecular structure of (PVBA) $)_{2} \mathrm{PbI}_{4}$ perovskite (d) and PVBA polymer configuration (e). The bending arrows represent the rotation direction of carbon-carbon bond. $\mathbf{f}, \mathrm{Pb}-\mathrm{I}-\mathrm{Pb}$ bond angles for $(\mathrm{VBA})_{2} \mathrm{PbI}_{4}$ and $(\mathrm{PVBA})_{2} \mathrm{PbI}_{4}$. 

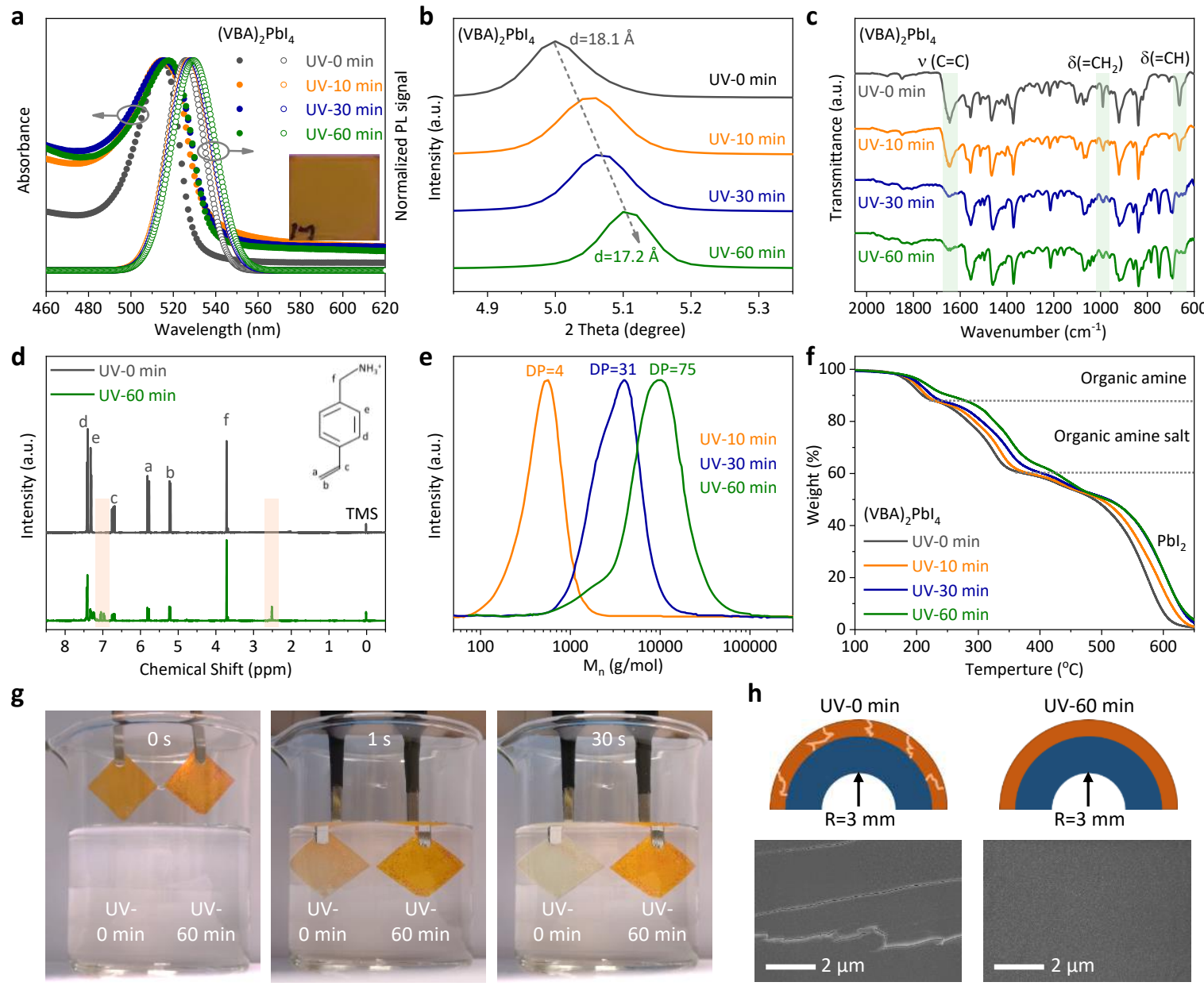

UV-60 $\mathrm{min}$

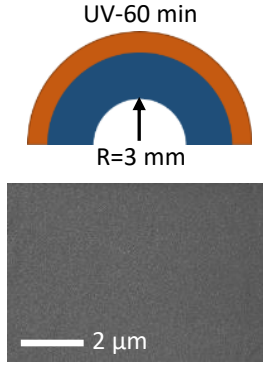

Fig. 2 | Experimental demonstration of the formation of polymerized perovskite and its

polymer-like behavior. a-f, Absorption and PL spectra (a), XRD patterns (b), FTIR spectra

(c), ${ }^{1} \mathrm{H}$ NMR spectra (d), GPC traces (e) and TGA curves (f) for (VBA) $)_{2} \mathrm{PbI}_{4}$ films with various

UV exposure durations. Inset in (a): A photograph of an as-prepared (VBA) ${ }_{2} \mathrm{PbI}_{4}$ film. g, Photographs of the (VBA) $)_{2} \mathrm{PbI}_{4}$ and $(\mathrm{PVBA})_{2} \mathrm{PbI}_{4}$ perovskite films immersed in water for

541 different durations. h, Schematic illustrations and corresponding SEM images of the

$542(\mathrm{VBA})_{2} \mathrm{PbI}_{4}$ and $(\mathrm{PVBA})_{2} \mathrm{PbI}_{4}$ perovskite films (thickness: $500 \mathrm{~nm}$ ) after 500 bending cycles

543 with a bending radius of $3 \mathrm{~mm}$. 

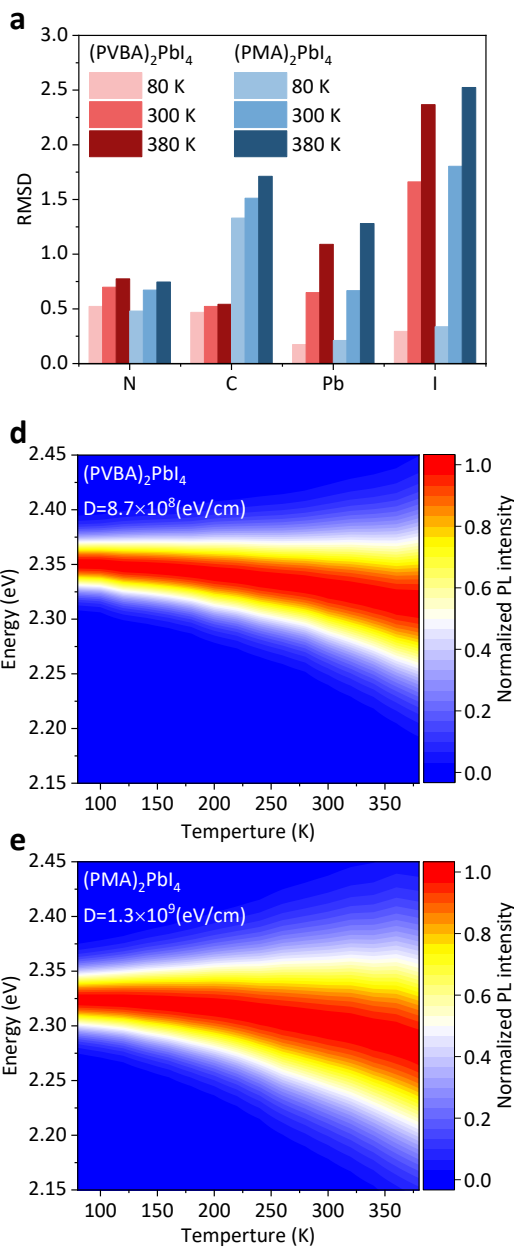

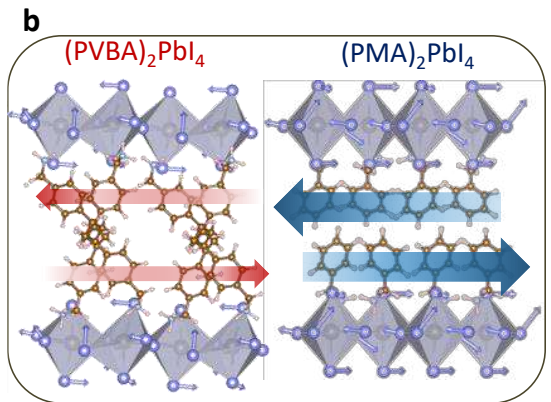

f
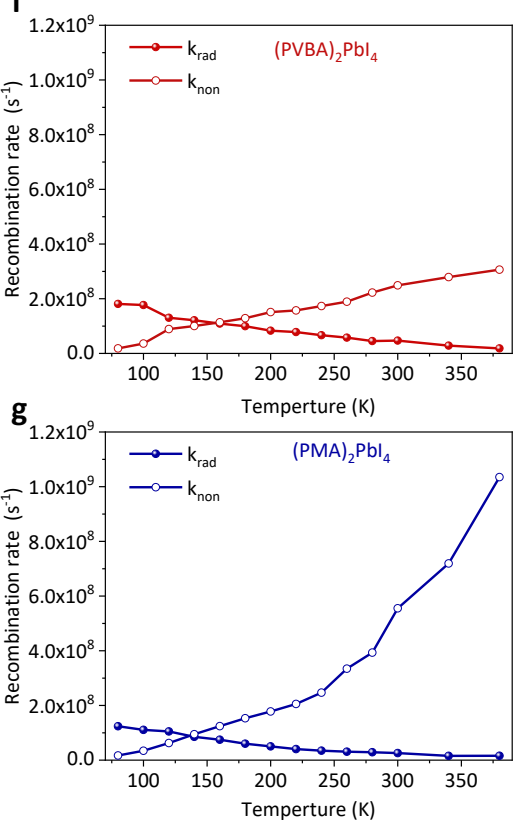

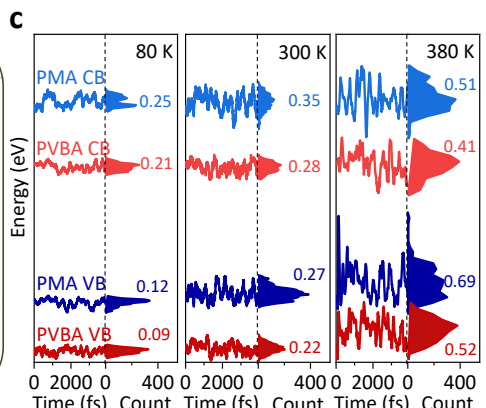
h

$$
\text { h }
$$
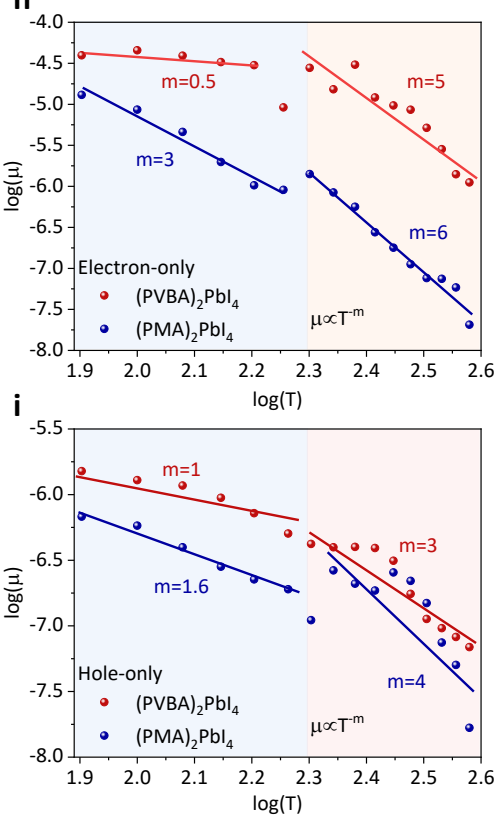

Fig. 3 | Enhanced rigidity of the polymerized perovskite and its influence on

optoelectronic properties. a, Average atomic displacement of $\mathrm{N}, \mathrm{C}, \mathrm{Pb}$ and $\mathrm{I}$ atoms of

547 (PVBA $)_{2} \mathrm{PbI}_{4}$ and $(\mathrm{PMA})_{2} \mathrm{PbI}_{4}$ from DFT calculation. b, Real-space displacement of the

548 dominated modes of $(\mathrm{PVBA})_{2} \mathrm{PbI}_{4}$ and $(\mathrm{PMA})_{2} \mathrm{PbI}_{4}$ structures. The arrow direction and

549 thickness represent direction and intensity of atom vibration, respectively. c, Bandedge

550 fluctuations and corresponding histograms for $(\mathrm{PVBA})_{2} \mathrm{PbI}_{4}$ and $(\mathrm{PMA})_{2} \mathrm{PbI}_{4}$. The numbers are

551 the FWHM values of the histograms. d-i, Temperature-dependent steady-state PL spectra (d,e),

$552 \mathrm{k}_{\mathrm{rad}}$ and $\mathrm{k}_{\text {non }}(\mathbf{f}, \mathbf{g})$, and electron/hole mobilities $(\mathbf{h}, \mathbf{i})$ of both $(\mathrm{PVBA})_{2} \mathrm{PbI}_{4}$ and $(\mathrm{PMA})_{2} \mathrm{PbI}_{4}$. 
a

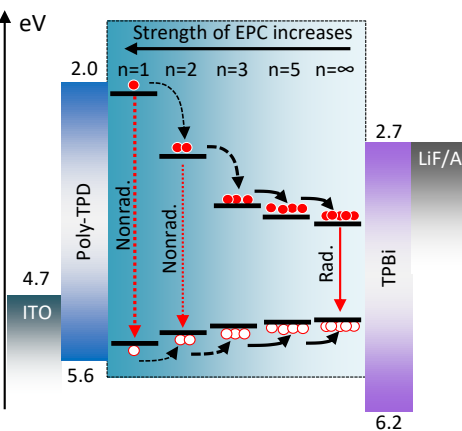

d

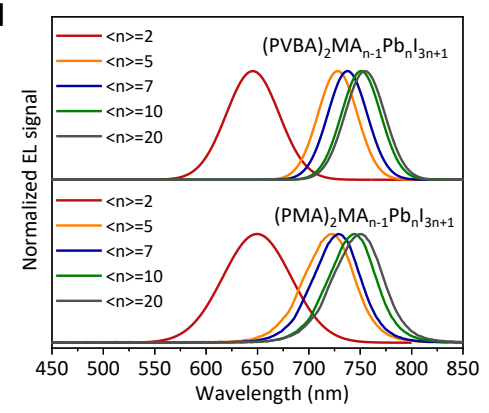

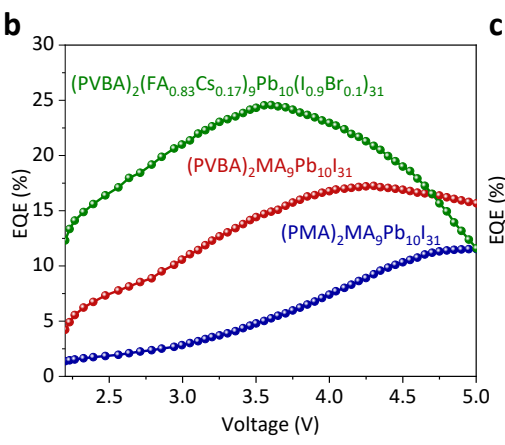
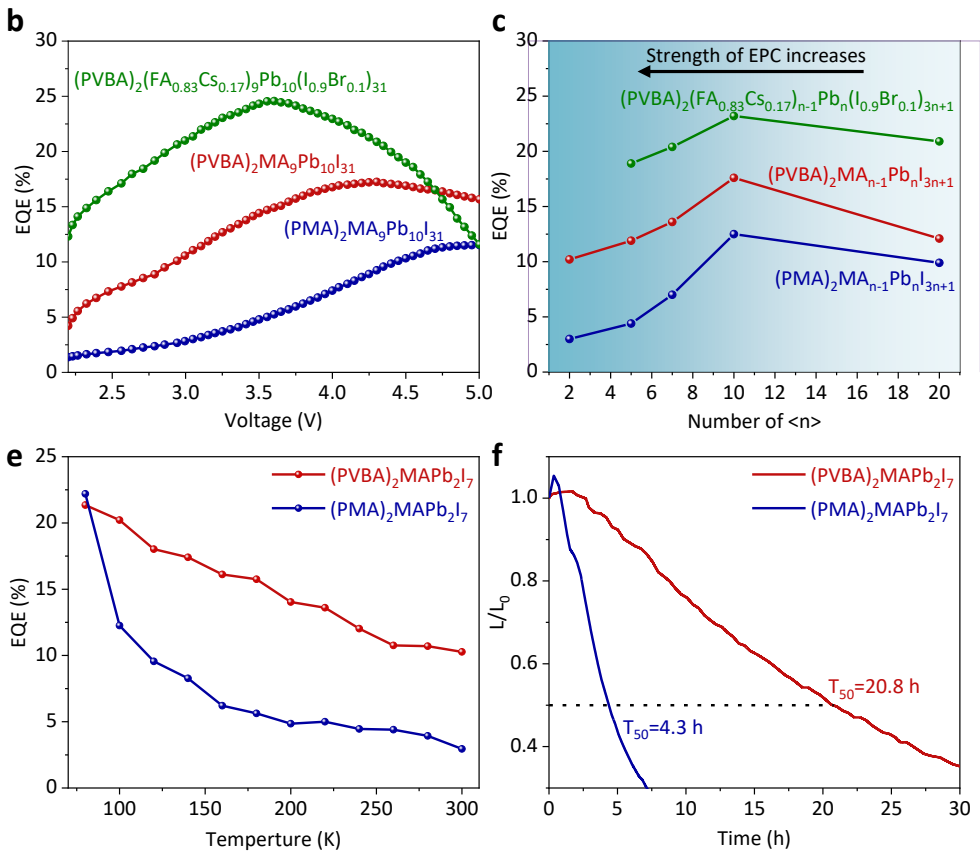

Fig. 4 | Device performances of polymerized PeLEDs. a, Energy diagram of quasi-2D

PeLEDs. Rad. and Nonrad. represent radiative recombination and non-radiative recombination,

respectively. b, EQE curves of the PeLEDs based on different quasi-2D perovskites. c,d, EQE

values (c) and EL spectra (d) of the quasi-2D PeLEDs with different $<\mathrm{n}>$ values. e,

Temperature-dependent peak EQE of (PVBA) ${ }_{2} \mathrm{MAPb}_{2} \mathrm{I}_{7}$ and $(\mathrm{PMA})_{2} \mathrm{MAPb}_{2} \mathrm{I}_{7}$ based PeLEDs.

f, Operation stability of the PeLEDs measured at a constant current density of $5 \mathrm{~mA} / \mathrm{cm}^{2}$. 
Figures

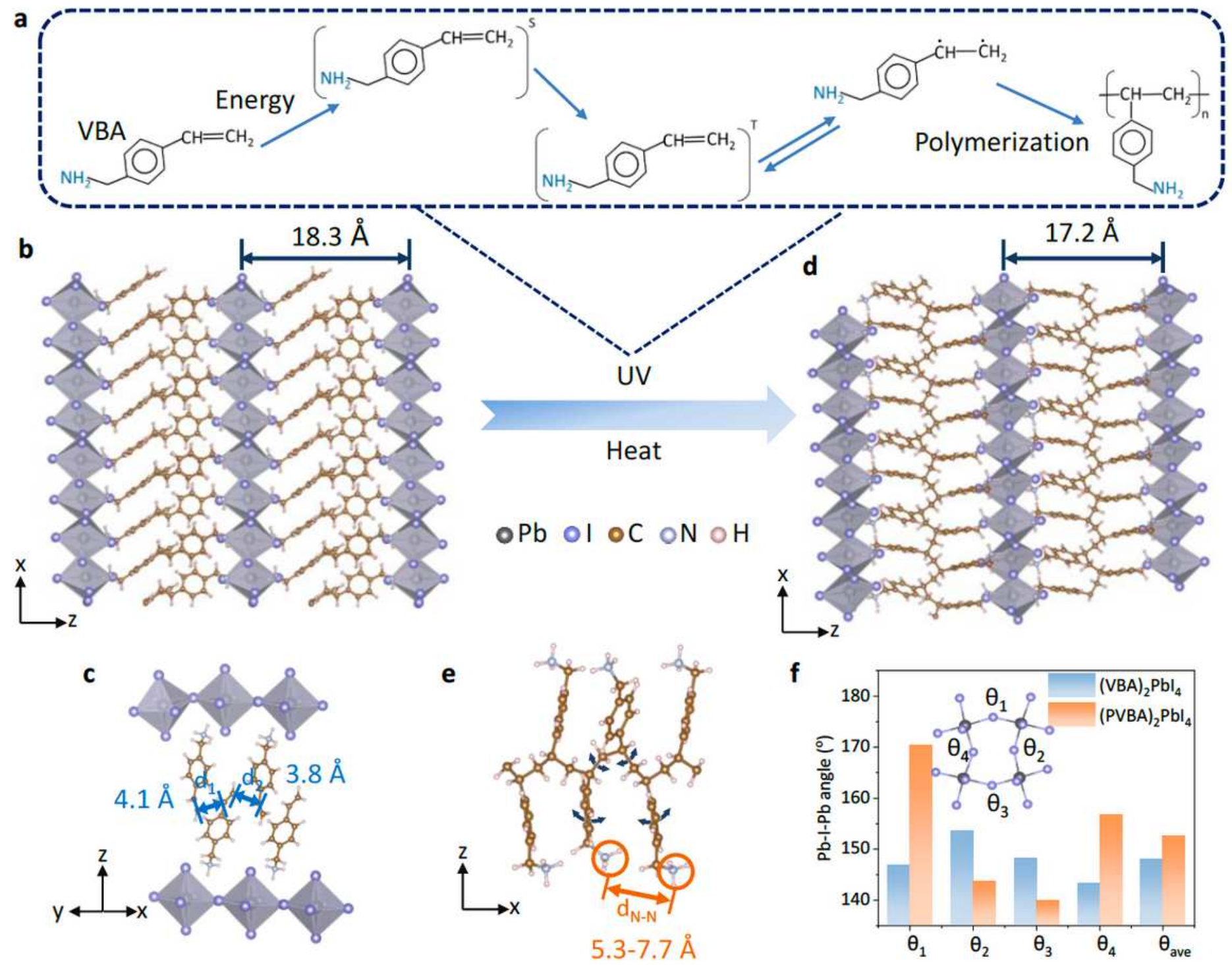

Figure 1

Theoretical calculation of polymerized perovskite structure. 

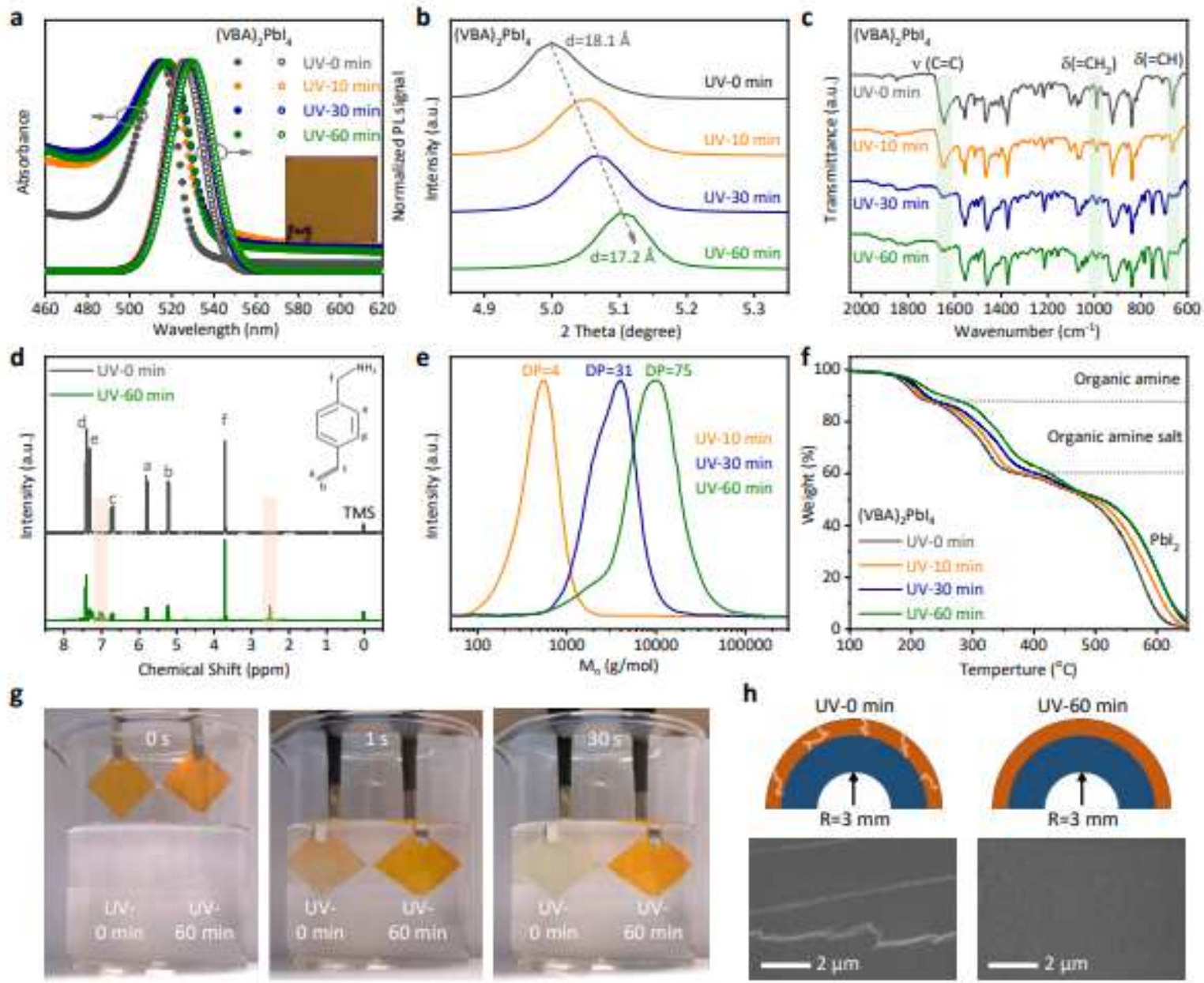

h
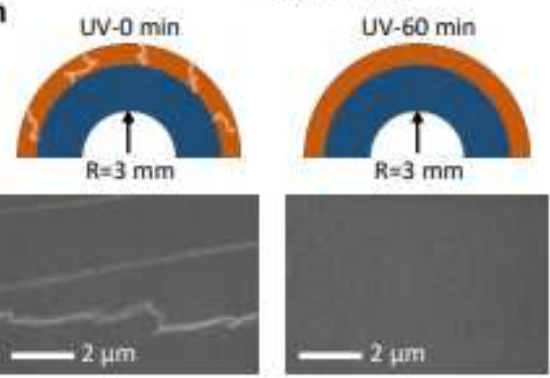

Figure 2

Experimental demonstration of the formation of polymerized perovskite and its polymer-like behavior. 

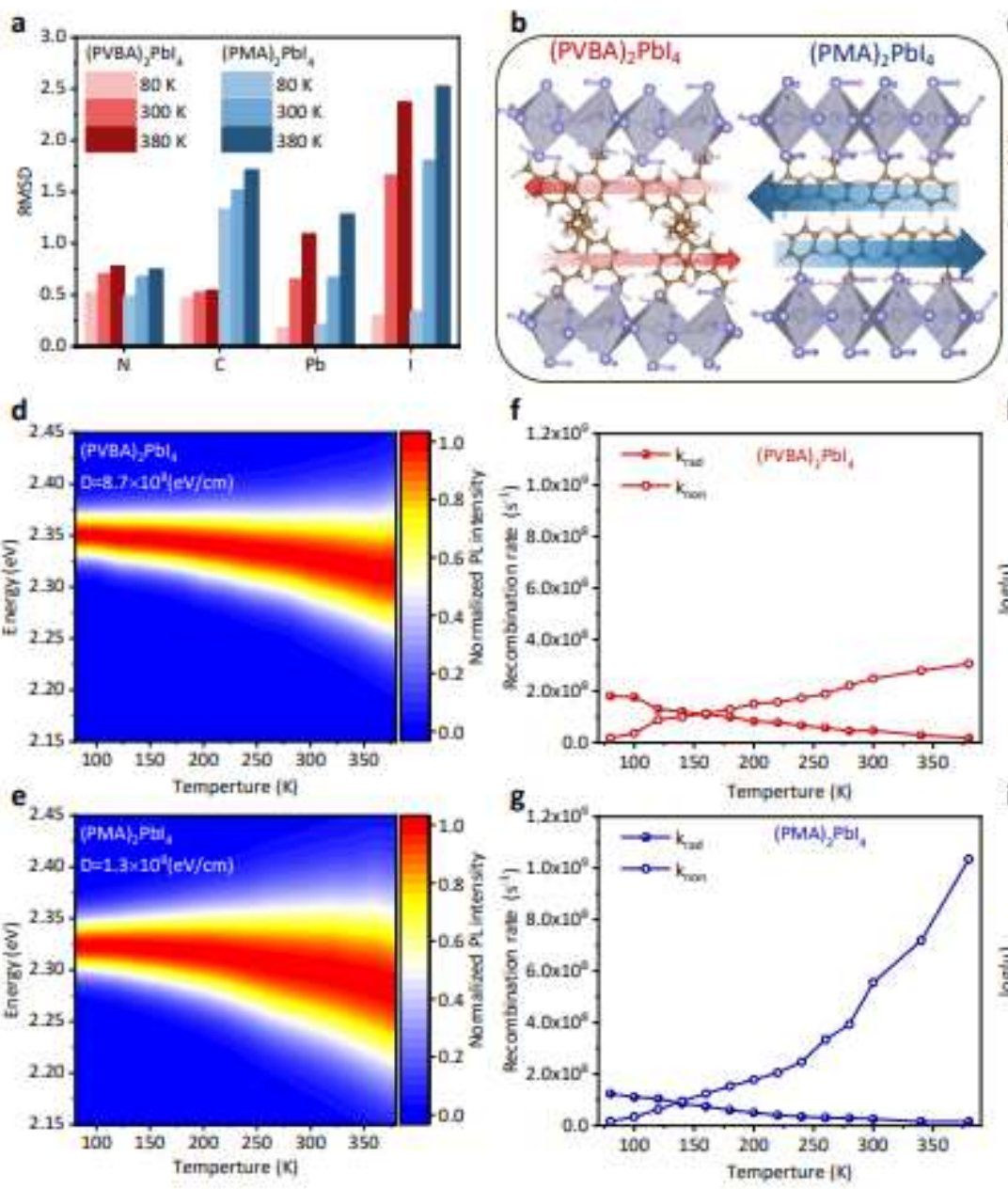

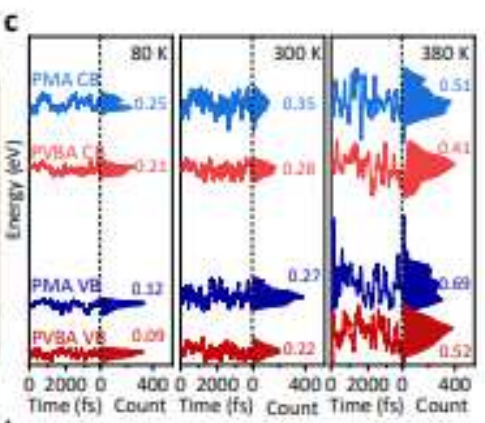

h
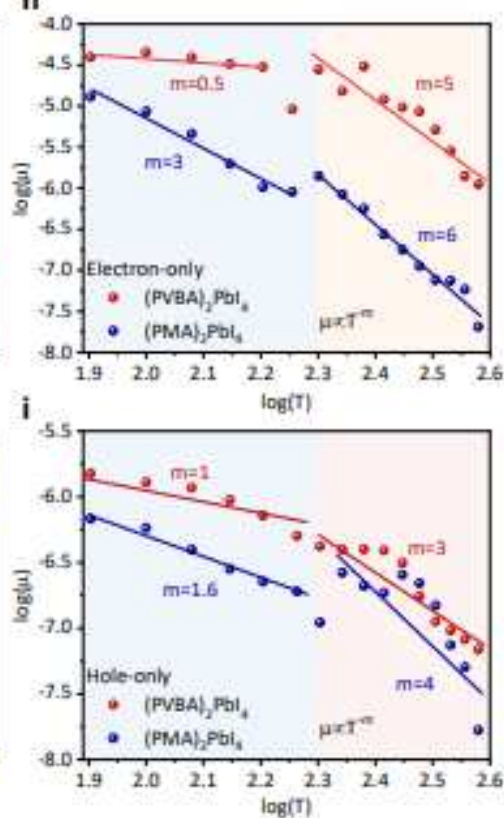

\section{Figure 3}

Enhanced rigidity of the polymerized perovskite and its influence on optoelectronic properties.

a
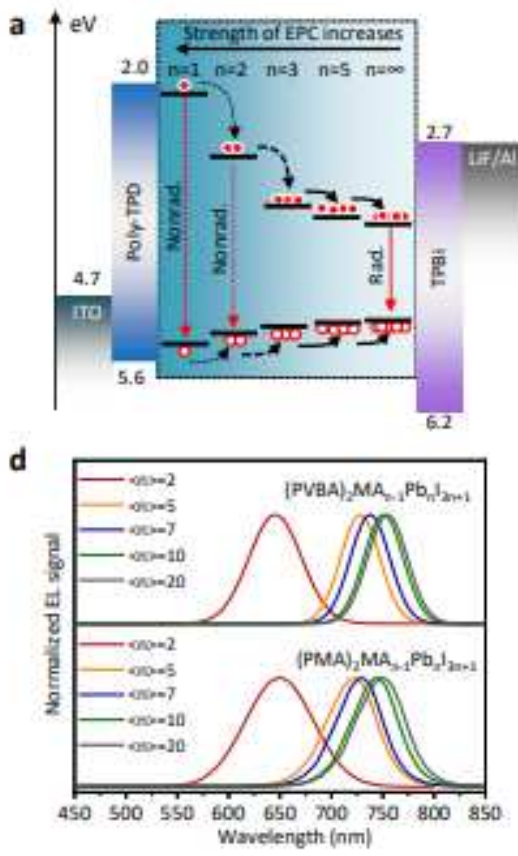

b
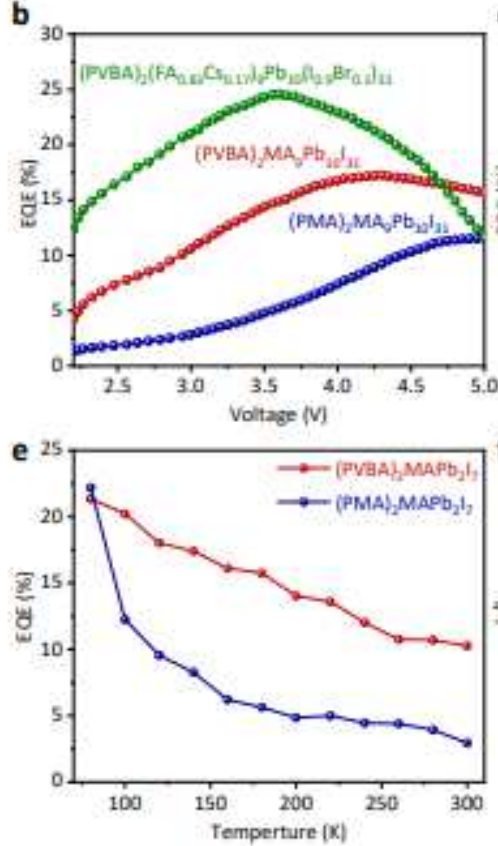
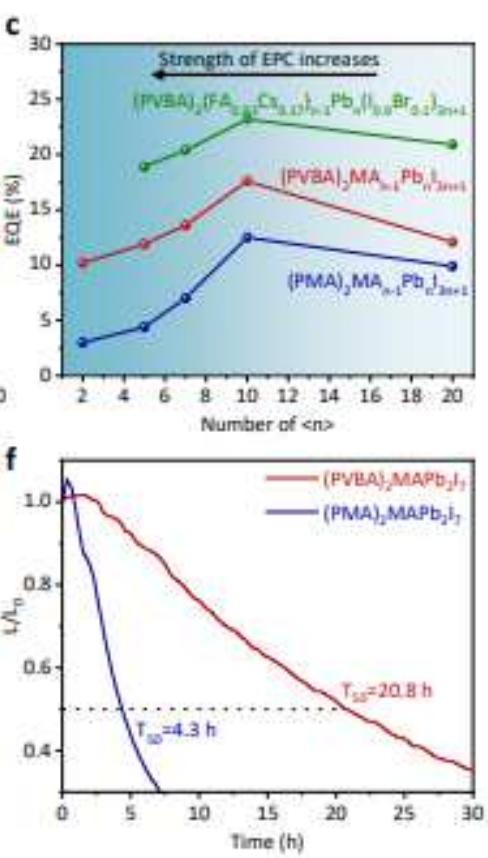
Figure 4

Device performances of polymerized PeLEDs.

\section{Supplementary Files}

This is a list of supplementary files associated with this preprint. Click to download.

- SupplementaryVideo.mov

- RevisedSupplementaryInformation.pdf 